\title{
Non-typeable Haemophilus influenzae and Streptococcus pneumoniae as primary causes of acute otitis media in colombian children: a prospective study
}

Alexandra Sierra', Pio Lopez ${ }^{1 *}$, Mercedes A Zapata', Beatriz Vanegas', Maria M Castrejon², Rodrigo DeAntonio², William P Hausdorff ${ }^{3}$, Romulo E Colindres ${ }^{4}$

\begin{abstract}
Background: Acute otitis media (AOM) is one of the most frequently encountered bacterial infections in children aged < 5 years; Streptococcus pneumoniae (S. pneumoniae) and non-typeable Haemophilus influenzae (NTHi) are historically identified as primary AOM causes. Nevertheless, recent data on bacterial pathogens causing AOM in Latin America are limited. This prospective study aimed to identify and characterize bacterial etiology and serotypes of AOM cases including antimicrobial susceptibility in $<5$ year old Colombian children.

Methods: From February 2008 to January 2009, children $\geq 3$ months and $<5$ years of age presenting with AOM and for whom a middle ear fluid (MEF) sample was available were enrolled in two medical centers in Cali, Colombia. MEF samples were collected either by tympanocentesis procedure or spontaneous otorrhea swab sampling. Bacteria were identified using standard laboratory methods, and antimicrobial resistance testing was performed based on the 2009 Clinical and Laboratory Standards Institute (CLSI) criteria. Most of the cases included in the study were sporadic in nature.

Results: Of the 106 enrolled children, 99 were included in the analysis. Bacteria were cultured from 62/99 (63\%) of samples with S. pneumoniae, H. influenzae, or S. pyogenes. The most commonly isolated bacteria were $H$. influenzae in 31/99 (31\%) and S. pneumoniae in 30/99 (30\%) of samples. The majority of H. influenzae episodes were NTHi (27/ 31; 87\%). 19F was the most frequently isolated pneumococcal serotype (10/30; 33\%). Of the $30 \mathrm{~S}$. pneumoniae positive samples, $8 / 30$ (27\%) were resistant to tetracycline, 5/30 (17\%) to erythromycin and 8/30 (27\%) had intermediate resistance to penicillin. All $H$. influenzae isolates tested were negative to beta-lactamase.
\end{abstract}

Conclusions: NTHi and S. pneumoniae are the leading causes of AOM in Colombian children. A pneumococcal conjugate vaccine that prevents both pathogens could be useful in maximizing protection against AOM.

\section{Background}

Acute otitis media (AOM) is one of the most frequent bacterial infections in infancy and early childhood worldwide. Though diagnosed in all ages, AOM is relatively common among children between six months to three years of age with approximately $80 \%$ of children having had at least one episode of AOM by the time they are three years old [1]. AOM is one of the main

\footnotetext{
* Correspondence: piolo@emcali.net.co

'Centro de Estudios en Infectologia Pediatrica CEIP, Cali, Colombia Full list of author information is available at the end of the article
}

causes of childhood morbidity in both developed and developing countries [2]. The estimated annual disease burden of AOM ranges between 8,200,000 and $12,900,000$ cases among children less than five years of age in Latin America and the Caribbean [3], compared to that in US children [4] where the estimated number of AOM per year are 10,200,000. In Europe, the high incidence of AOM during childhood has been confirmed, estimated to range from 17,600 to 38,700 episodes per 100,000 person-years in children under five years of age [5].

\section{C) Biomed Central}


While both bacteria and/or viruses can cause AOM, the most serious infections are believed to involve bacterial pathogens [6], particularly Streptococcus pneumoniae (S. pneumoniae) or non-typeable Haemophilus influenzae (NTHi), which together account for at least 60-70\% of clinical AOM episodes [7]. Other less frequently reported bacterial agents of AOM are Moraxella catarrhalis (M. catarrhalis), Streptococcus pyogenes (S. pyogenes) and Staphylococcus aureus (S. aureus) [8,9]. Limited studies from Latin America have suggested that S. pneumoniae is the most common bacterial pathogen found in middle ear fluid samples from AOM cases $[10,11]$. However, in recent years, Haemophilus influenzae (H. influenzae) is increasingly being recognized as a prominent bacterial pathogen in AOM $[11,12]$. In addition, many of the AOM etiology studies referred to above are centered on treatment failure or persistent AOM patients and not sporadic cases even though the latter represent the vast majority of AOM, and thus, even the global data may not reflect the true etiology of the most common form of AOM [13].

Although some AOM cases resolve spontaneously, the majority receives antibiotic treatment in order to diminish the duration of symptoms and increase the likelihood of resolution [14]; AOM is one of the primary reasons for pediatricians to prescribe antibiotics [15-17]. Constant use of antibiotics over the years has led to an increase in resistance/non-susceptibility to antibiotics of the bacterial pathogens. Some of the antibiotic-resistant serotypes of $S$. pneumoniae are the ones that are most often involved in serious pneumococcal infections [18]. In Latin America, the serotypes $6 \mathrm{~A}, 6 \mathrm{~B}, 9 \mathrm{~V}, 14,19 \mathrm{~A}, 19 \mathrm{~F}$, and $23 \mathrm{~F}$ are responsible for most of the antibiotic-resistant

$S$. pneumoniae infections that cause invasive disease in children [19]. According to the Surveillance reports of the Regional System for Vaccines (SIREVA), coordinated by the Microbiology Group of the National Institute of Health of Colombia, of the 90 different serotypes of S. pneumoniae identified so far, serotype 14 is the most frequently isolated serotype in Colombia for invasive pneumococcal disease [20].

Widespread immunization of children with pneumococcal conjugate vaccines might play a role in reducing AOM episodes. The heptavalent pneumococcal conjugate vaccine Prevnar $^{\mathrm{Tm}} /$ Prevenar $^{\mathrm{Tm}}$, Pfizer/Wyeth, USA; PCV-7) was introduced as public immunization for US children in 2000. In clinical efficacy trials, this vaccine had previously been shown to have $57 \%$ efficacy against vaccine-type pneumococcal AOM [21], and overall 7\% efficacy against clinical AOM episodes [22]. With the use of antibiotics at high doses and PCV-7, there has been a change in the pneumococcal serotypes responsible for persistent AOM and an increasing predominance of $H$. influenzae [23,24].
In the recently licensed 10 -valent pneumococcal $/ H$. influenzae protein D conjugate vaccine (Synflorix ${ }^{\mathrm{Tm}}$, GlaxoSmithKline [GSK] Biologicals, Rixensart, Belgium; PHiD-CV) that contains the same serotypes as found in PCV-7 plus the invasive serotypes 1,5 , and $7 F, 8$ of the 10 serotypes are conjugated to a recombinant form of protein D of non-typeable $H$. influenzae. A randomized, double-blind, controlled study with a prototype 11 -valent protein $\mathrm{D}$ formulation suggests that $\mathrm{PHiD}-\mathrm{CV}$ may provide similar protection against pneumococcal AOM as that seen with PCV7 and additional protection (35\%) against episodes of AOM caused by NTHi [25]. A 13 -valent pneumococcal vaccine formulation (PCV-13) is yet to be licensed in Colombia.

Current data on etiology of AOM in Colombian children are limited and scarce, with one study on Colombian children with AOM conducted over 20 years ago [6]. After the 7-valent pneumococcal conjugate vaccine was introduced in the Colombian private markets in early 2002, it remains important to characterize the bacteria responsible for AOM and to evaluate the potential impact and effectiveness of vaccination with conjugate vaccines against $H$. influenzae and/or pneumococcal AOM. This prospective study aims to identify and characterize bacterial etiology and serotypes including antimicrobial susceptibility of AOM in Colombian children less than five years of age.

\section{Methods}

\section{Study design and subjects}

This is a prospective epidemiological study conducted in routine clinical setting at two medical centers in Cali, Colombia. Children between three months and five years of age, visiting two pediatric clinics for AOM from February 2008 to January 2009, and for whom a middle ear fluid (MEF) sample was available were enrolled in the study.

The study included children visiting the pediatrician with one of the general signs for AOM (otalgia/irritability, conjunctivitis, fever and either Paradise's criteria (bulging, diffused, or localized inflamed tympanic membranes) or spontaneous otorrhea (less than 24 hours). Patients identified for recruitment were either children with a new episode of AOM (less than 72 hours of onset) who had not yet received antibiotics for the episode (untreated group), or children who had a diagnosis of AOM within 48-72 hours prior to study enrolment, and who received antibiotic therapy from a physician but remained symptomatic at the time of study entry (treatment failures). Children who received systemic antibiotic treatment for a disease other than AOM in the 72 hours prior to enrolment and ones receiving antimicrobial prophylaxis for recurrent AOM were excluded. Recurrent AOM was defined as $\geq 3$ episodes 
in the past 6 months or $\geq 4$ episodes in the past 12 months. There were no restrictions on antibiotic use following tympanocentesis. All children were treated according to local practices.

The primary endpoints were occurrence of $H$. influenzae, $S$. pneumoniae and other bacterial pathogens isolated from MEF samples. Secondary endpoints were occurrence of $H$. influenzae and S. pneumoniae specific serotypes.

Written informed consent was obtained from the parent/guardian of the child before conducting any studyrelated procedures. Demographic information and child's medical history, case history and general symptoms were collected and a clinical examination was performed.

The study included only sporadic cases once they received attention at the study sites using standard criteria accepted internationally for AOM and using standard methods to detect bacterial agents avoiding selection bias.

\section{Bacterial identification and characterization}

Samples of MEF for all children were collected by an ENT specialist by tympanocentesis or by careful sampling of spontaneous otorrhea for children who had spontaneous/accidental rupture of the tympanic membrane. The latter entailed removing and cleaning the ear canal by deep aspiration of the MEF material through the perforation to minimize contamination and spurious results. For the purposes of this study, only S. pneumoniae, NTHi, S. pyogenes and M. catarrhalis were considered true pathogens [26].

Inoculation of samples was done into Amies transport medium and the sample was plated at the GSK designed laboratory within the first 48 hours at room temperature for further analysis. Standard methodology was employed in order to determine the antimicrobial susceptibility. The cut-offs for susceptibility and resistances are provided in Table 1 . The samples were coded with the identification number for the child.

\section{Statistical analyses}

The analysis was performed on the According-To-Protocol (ATP) cohort (evaluable subjects meeting all eligibility criteria, complied with the protocol defined procedures, with no elimination criteria during the study and for whom laboratory results of the middle ear fluid sample were available). A recurrent AOM episode was the third or more episode in the last six months or the fourth or more episode in the last 12 months.

The proportions of AOM caused by $S$. pneumoniae, $H$. influenzae and other bacterial pathogens were calculated with their $95 \%$ confidence interval (CI). Distribution analysis of S. pneumoniae and $H$. influenzae serotypes
Table 1 Ranges for S. pneumoniae and H. influenzae antimicrobial susceptibility ote: of companies theentioned in the results now. 36-47 months age group. ren: Potential implications for pneumococcal con

\begin{tabular}{|c|c|c|}
\hline Antibiotic & $\begin{array}{l}\text { Susceptible }(S)(\mu \mathrm{g} / \\
\mathrm{mL})\end{array}$ & $\begin{array}{c}\text { Resistant (R) }(\mu \mathrm{g} / \\
\mathrm{mL})\end{array}$ \\
\hline \multicolumn{3}{|l|}{ S. pneumoniae } \\
\hline Penicillin & $\leq 0.06$ & $\geq 2$ \\
\hline Cefotaxime & $\leq 0.06$ & $\geq 4$ \\
\hline Ceftriaxone & $\leq 0.06$ & $\geq 4$ \\
\hline Chloramphenicol & $\leq 2$ & $\geq 32$ \\
\hline Erythromycin & $\leq 0.06$ & $\geq 1$ \\
\hline Levofloxacin & $\leq 0.5$ & $\geq 8$ \\
\hline Linezolid & $\leq 2$ & $\geq 4$ \\
\hline Moxifloxacin & $\leq 0.25$ & $\geq 4$ \\
\hline Ofloxacin & $\leq 1$ & $\geq 8$ \\
\hline Tetracycline & $\leq 1$ & $\geq 16$ \\
\hline $\begin{array}{l}\text { Trimethoprim/ } \\
\text { sulfamethoxazole }\end{array}$ & $\leq 0.5 / 9.5$ & $\geq 16 / 304$ \\
\hline Vancomycin & $\leq 1$ & $\geq 2$ \\
\hline \multicolumn{3}{|l|}{ H. influenzae } \\
\hline Ampicillin & $\leq 1$ & $>1$ \\
\hline Amoxicillin-clavulanate & $\leq 1$ & $>1$ \\
\hline Cefotaxime & $\leq 0.12$ & $>0.12$ \\
\hline Cefuroxime & $\leq 1$ & $>2$ \\
\hline Cefuroxime axetil & $\leq 0.12$ & $>1$ \\
\hline Levofloxacin & $\leq 1$ & $>1$ \\
\hline Erythromycin & $\leq 0.5$ & $>16$ \\
\hline Tetracycline & $\leq 1$ & $>2$ \\
\hline Chloramphenicol & $\leq 1$ & $>2$ \\
\hline $\begin{array}{l}\text { Trimethoprim- } \\
\text { sulfamethoxazole }\end{array}$ & $\leq 0.5$ & $>1$ \\
\hline
\end{tabular}

Source: CLSI 2008 - M100-S19- PAGE 65 and 66

by age, by procedure and by pneumococcal vaccination status for AOM cases was performed. Seasonality of AOM cases was also determined.

All statistical analyses were performed using statistical analysis system (SAS) version 9.1 and Microsoft Excel (for graphical purposes).

\section{Ethics}

The study was conducted according to Good Clinical Practice guidelines, the Declaration of Helsinki and the local rules and regulations of the country. All the studyrelated documents were reviewed and approved by the local Independent Ethics Committee and the study adhered to applicable local guidelines.

\section{Results}

\section{Demography}

During the 12-month period, a total of 106 (103 untreated and 3 treatment failures) children were enrolled; all reporting one episode each. Of these, seven 
children were eliminated from the ATP cohort: two children (treatment failure) received medication (amoxicillin and cefalexin) forbidden in the protocol, one child (treatment failure) had an onset of signs and symptoms of AOM beyond 72 hours of diagnosis, MEF samples could not be collected from three cases, and one child was enrolled twice for the same episode (elimination at the second episode). All 99 (93\%) children included in the ATP analyses were untreated with antibiotics; of these, seven cases were classified as recurrent AOM episodes. One child had a bilateral episode. No treatment failures were included in the ATP cohort since none met the enrolment criteria.

The mean age of children was 28.5 months and 54/99 (55\%) were males (Table 2). The highest percentage of episodes was reported among children 12-23 months and 24-35 months of age 27/99 (27\% each). No statistically significant differences of pathogen distribution by demographic characteristics were noted. Majority of the episodes were positive for at least one S. pneumoniae, $H$. influenzae or S. pyogenes among children 3-11 months (9/11, 82\%), 12-23 months $17 / 27$ (63\%), and 24-35 months $(18 / 27 ; 67 \%)$ of age. $H$. influenzae was isolated in all age groups described for this study, $45 \%$ (14/31) of these isolates corresponded to children under 2 years of age and reporting the same number of cases in children 3-11 and 12-23 months (n = 7). On the other hand, $S$. pneumoniae was not isolated in children 48-59 months. Among these isolates, 40\% (12/30) occurred in children less than 24 months of age, distributed as 3 isolates in children 3-11 (10\%) and 9 in 12-23 months (30\%).

More females were positive $(31 / 45,69 \%)$ for at least one study bacteria than males $(30 / 54,56 \%)$. No statistically significant differences of pathogen distribution by gender characteristics were identified. Of the seven recurrent cases, four (15\%) belonged to the 36-47 months age group.

\section{Seasonality}

AOM episodes occurred throughout the year; however, the maximum number of cases was enrolled between February and April 2008 with a smaller peak occurring in August 2008 corresponding to the rainy season in Cali. H. influenzae was isolated during all months, with higher prevalence in August $(\mathrm{n}=7)$, February $(\mathrm{n}=5)$, and March $(\mathrm{n}=5)$. S. pneumoniae was predominantly isolated in February $(n=7)$ and June $(n=5)$, but also found throughout the year (Figure 1).

\section{Sample Collection}

Only one MEF sample was collected from 98/99 AOM untreated episodes (one episode had two samples collected, one from each ear). Of the 99 untreated episodes,
$84 / 99(85 \%)$ of samples were collected by tympanocentesis and 16/99 (16\%) by spontaneous otorrhea.

\section{Bacterial etiology}

Pathogenic bacteria were cultured from 62/99 (63\%) of episodes with the bacterium: S. pneumoniae, H. influenzae, or S. pyogenes. Over 13/16 (81\%) of episodes collected by otorrhea swab were bacterial positive, compared to $49 / 83$ (59\%) collected by tympanocentesis. Overall, 68 bacterial agents were isolated, corresponding to 56 episodes with a single pathogen and 6 episodes with 2 pathogens. The most commonly isolated bacteria were $H$. influenzae in $31 / 99$ (31\%) and S. pneumoniae in $30 / 99$ (30\%) of episodes, with S. pyogenes representing only $2 / 99(2 \%)$ of cases and other bacteria in $5 / 99$ (5\%) cases (Figure 2). Samples collected from two children were positive for both $S$. pneumoniae and $H$. influenzae. No samples tested positive for $M$. catarrhalis. Most of $H$. influenzae and S. pneumoniae isolates were taken by tympanocentesis $(77 \%$ and $83 \%$, respectively). From 83 samples collected by tympanocentesis, $30.1 \%$ isolated $S$. pneumoniae and $28.9 \%$ isolated $H$. influenzae; and from 16 samples collected by otorrhea, $43.8 \%$ corresponded to $H$. influenzae and $31.3 \%$ to S. pneumoniae. H. influenzae was isolated in 6/7 recurrent AOM episodes, meanwhile $S$. pneumoniae was isolated in only $1 / 7$ recurrent AOM.

At least one dose of the pneumococcal vaccine was received by $29 / 99$ (29\%) of children. The percentage of children who received at least one dose of heptavalent pneumococcal conjugate vaccine (PCV-7) was $30 \%$ (9/30) include numbers as for $H$ influenza bleow) in children who were culture positive for S. pneumoniae (seven fully vaccinated and two partially vaccinated) and $35 \%$ who were culture positive for $H$. influenzae (11/31).

\section{Serotype distribution of $H$. influenzae and S. pneumoniae} The majority of $H$. influenzae isolated were non-typeable (27/31; 87\%). NTHi was isolated from all $H$ influenzae episodes in the 3-11 months, 12-23 months and 48-59 months age groups. In children under two years of age, all isolates corresponded to being non-typeable, meanwhile, 4/17 (24\%) encapsulated isolates were identified in the 24-60 months age groups corresponding to $\mathrm{f}$ and b serotypes; the remaining 2 isolates were unknown.

Serotype 19F was the most frequently isolated pneumococcal serotype followed by $6 \mathrm{~A}$ and 14 . Serotypes 3 and 19A were the fourth most prevalent (Figure 3). The majority of the serotypes (3, 6A, 14, 16F, 19F, 20, 23F) were isolated in the 12-23 months age group. In fully vaccinated children, serotypes $19 \mathrm{~F}(\mathrm{n}=4)$, 19A $(\mathrm{n}=2)$ and $6 \mathrm{~A}(\mathrm{n}=1)$ were isolated. Serotypes $6 \mathrm{~A}$ and 3 were isolated in the partially vaccinated children. 
Table 2 Demographic characteristics with bacterial etiology of episodes by age and gender (ATP cohort)

\begin{tabular}{|c|c|c|c|}
\hline AOM Episodes Characteristics & Categories & Value or $n$ & $\%$ \\
\hline Age (months) & & & - \\
\hline Mean & & 28.5 & - \\
\hline SD & & 13.03 & - \\
\hline Median & & 29 & - \\
\hline Range & & $5-55$ & - \\
\hline \multicolumn{4}{|l|}{ Age group (months) } \\
\hline \multirow[t]{5}{*}{$0-11^{a}$} & & 11 & $11.1 \%$ \\
\hline & H. influenzae & 7 & - \\
\hline & S. pneumoniae & 3 & \\
\hline & S. pyogenes & 0 & - \\
\hline & Others/Negative & 5 & - \\
\hline \multirow[t]{5}{*}{$12-23^{b}$} & & 27 & $27.3 \%$ \\
\hline & H. influenzae & 7 & - \\
\hline & S. pneumoniae & 9 & - \\
\hline & S. pyogenes & 1 & - \\
\hline & Others/Negative & 11 & - \\
\hline \multirow[t]{5}{*}{$24-35$} & & 27 & $27.3 \%$ \\
\hline & H. influenzae & 5 & - \\
\hline & S. pneumoniae & 12 & - \\
\hline & S. pyogenes & 1 & - \\
\hline & Others/Negative & 9 & - \\
\hline \multirow[t]{5}{*}{$36-47^{c}$} & & 26 & $26.3 \%$ \\
\hline & H. influenzae & 8 & - \\
\hline & S. pneumoniae & 6 & - \\
\hline & S. pyogenes & 0 & \\
\hline & Others/Negative & 13 & - \\
\hline \multirow[t]{5}{*}{$48-59$} & & 8 & $8.1 \%$ \\
\hline & H. influenzae & 4 & - \\
\hline & S. pneumoniae & 0 & \\
\hline & S. pyogenes & 0 & - \\
\hline & Others/Negative & 4 & - \\
\hline \multicolumn{4}{|l|}{ Gender } \\
\hline \multirow[t]{5}{*}{ Female $^{d}$} & & 45 & $45.5 \%$ \\
\hline & H. influenzae & 17 & - \\
\hline & S. pneumoniae & 14 & - \\
\hline & S. pyogenes & 1 & - \\
\hline & Others/Negative & 17 & - \\
\hline \multirow[t]{5}{*}{ Male $^{\mathrm{e}}$} & & 54 & $54.5 \%$ \\
\hline & H. influenzae & 14 & - \\
\hline & S. pneumoniae & 16 & - \\
\hline & S. pyogenes & 1 & - \\
\hline & Others/Negative & 25 & - \\
\hline
\end{tabular}

$\mathrm{N}=99$; number of episodes.

$\mathrm{n}=$ number of episodes in a given category.

$\%=\mathrm{n} /$ Number of samples with results available $* 100$.

alt includes 3 episodes with more than 1 bacterial isolate (S. pneumoniae + H. influenzae+others; S. pneumoniae + others; H. influenzae + others).

${ }^{b}$ It includes 1 episode with more than 1 bacterial isolate (S. pneumoniae + others).

cIt includes 1 episode with more than 1 bacterial isolate (S. pneumoniae $+H$. influenzae).

${ }^{d}$ It includes 3 episodes with more than 1 bacterial isolate (S. pneumoniae $+H$. influenzae+others; S. pneumoniae + others; H. influenzae + others).

${ }^{\mathrm{e}}$ It includes 2 episodes with more than 1 bacterial isolate (S. pneumoniae $+H$. influenzae; $S$. pneumoniae + others). 


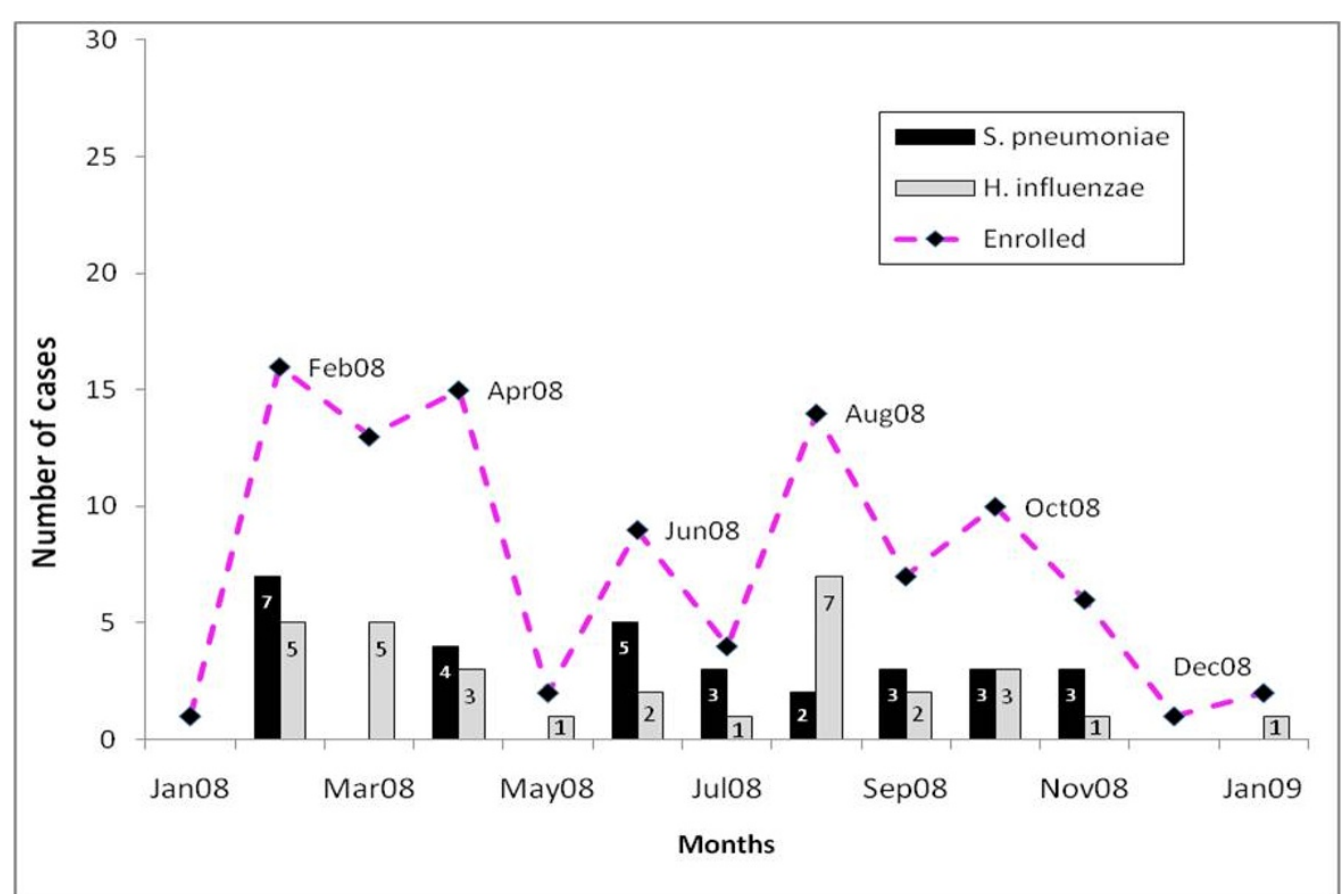

Figure 1 Seasonal distribution of AOM episodes (ATP cohort). The stacked line graph shows the number of episodes enrolled in each month and the simple bar graph shows the number of S. pneumoniae and $H$. influenzae isolated each month.

\section{Antibacterial susceptibility}

The prevalence of sensitive, intermediate and resistant strains for penicillin, among the $30 \mathrm{~S}$. pneumoniae positive samples from episodes, were $21 / 30$ (70\%), 8/30 (27\%) and $1 / 30(3 \%)$, respectively (Figure 4$)$. The strains resistant to other antibiotics were: $1 / 30(3 \%)$ to cefotaxime, $5 / 30(17 \%)$ to erythromycin and $8 / 30$ (27\%) to tetracycline and sulfamethoxazole/trimethoprim (Table 3). From S. pneumoniae isolates, serotype $19 \mathrm{~F}$ evidenced antibiotic resistance to tetracycline $(50 \% ; 5 / 10)$, trimethoprim/sulfamethoxazole $(30 \% ; 3 / 10)$ and erythromycin $(20 \% ; 2 / 20)$; serotype $19 \mathrm{~A}$ was resistant to penicillin, cefotaxime, tetracycline and erythromycin (50\%; $n=1 / 2)$, respectively.

All $H$. influenzae $(\mathrm{n}=31)$ tested with ampicillin were sensitive. From these isolates, 30 were tested for nitrocefin (beta-lactamase test) and reported as negative. All $H$. influenzae samples tested for penicillin (11/11) and for amoxicillin/clavulanate (26/26) were sensitive.

\section{Discussion}

Historically, the major bacteria responsible for most cases of AOM microbiology worldwide does not appear to have changed significantly over time, but the relative prevalence of main causal agents have changed in recent years [9]. In this study, approximately $63 \%$ of the samples cultured pathogenic bacteria. This proportion is in line with that seen in another study [27].
H. influenzae and S. pneumoniae are identified as the main causative agents of AOM in the current study, accounting for $31 \%$ and $30 \%$ of episodes, respectively. These results are similar to findings from other studies conducted in Latin American countries including Argentina [28], Chile [11], Costa Rica [10,29] and Mexico [30] where the leading causes of bacterial AOM were $S$. pneumoniae and H. influenzae. The studies in Argentina, Chile and Mexico looked at sporadic cases; this study shows that major pathogens in sporadic cases are similar to the mentioned studies. Increasing recognition of $H$. influenzae overtaking S. pneumoniae as the most frequent cause of AOM has recently been observed [9]. A previous study in the United States (1995-2003) assessed the changes in frequency and pathogens causing AOM since introduction of PCV-7 and reported a significant decrease in S. pneumoniae and an increase in $H$. influenzae among MEF isolates suggesting that $H$. influenzae has become the predominant pathogen of AOM since the inclusion of pneumococcal conjugate vaccine in the universal immunization program $[13,23]$. No significant differences in culture positivity by demographic characteristics and pneumococcal vaccination status were noted in this study with $66 \%$ of vaccinated and $61 \%$ of unvaccinated children being culture positive for at least one bacteria. However, vaccinated children showed a trend towards non-typeable $H$. influenzae (35\%) compared to S. pneumoniae (30\%). 


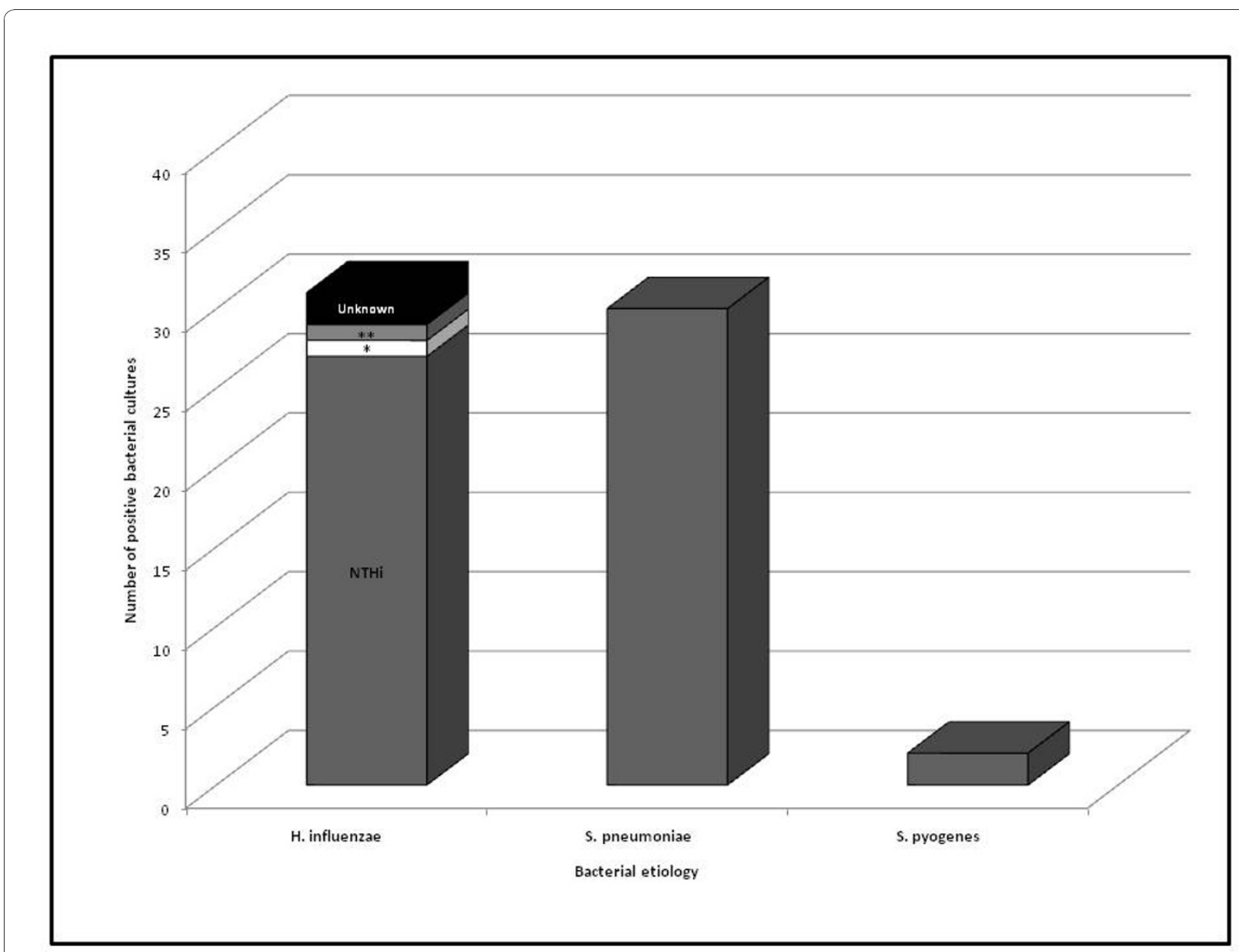

Figure 2 Etiology of bacteria identified from samples cultured from middle ear fluid. Number of positive bacterial episodes is represented in the stacked column bar. The bar for $\mathrm{H}$. influenzae also includes serotypes of $H$. influenzae positive episodes. ${ }^{*} H$. influenzae type $b{ }^{* *} H$. influenzae type $f$

In this study, H. influenzae was detected in all age groups, including during the first two years of life. This finding evidences the major role of this pathogen not only in older subjects as have been previously reported, but also in the younger children where the prominence of $H$. influenzae was not expected. Also, $H$. influenzae was present in most of the samples collected by otorrhea which is opposite from the findings in previous reports where most of otorrhea samples corresponded to S. pneumoniae. NTHi was responsible for $27 / 31$ (87\%) H. influenzae AOM episodes in this study. NTHi has shown to account for more recurrent AOM compared to S. pneumoniae and S. pyogenes and is associated with a history of recurrent AOM episodes, treatment failure and AOM within two weeks of completing a course of any antibiotic [31], making it a difficult-to-treat pathogen. This was confirmed in this study also where 6/7 recurrent AOM cases were due to NTHi.
Similar to previous reports, M. catarrhalis and S. pyogenes were not major pathogens in this study $[28,32,33]$.

Increase of resistance of $S$. pneumoniae to penicillin and other antibiotics is a concern in Latin America [34]. However, the current study shows limited prevalence of penicillin resistant S. pneumoniae but 8/30 (27\%) had intermediate resistance to penicillin. Consistent with the findings in Colombia [6], Costa Rica [10] and USA [35], all $H$. influenzae strains tested were beta-lactamasenegative in this study suggesting that both amoxicillin as well as amoxicillin/clavulanate could be considered as an AOM treatment option [36].

From the reported pneumococcal serotypes in this study, $19 \mathrm{~F}$ was the most frequently isolated pneumococcal serotype in this study, followed by $6 \mathrm{~A}$ and 14 . That four $19 \mathrm{~F}$ cases were detected in fully vaccinated children appears consistent with the results of the PCV7 AOM efficacy trial conducted in Finland, where the lowest 


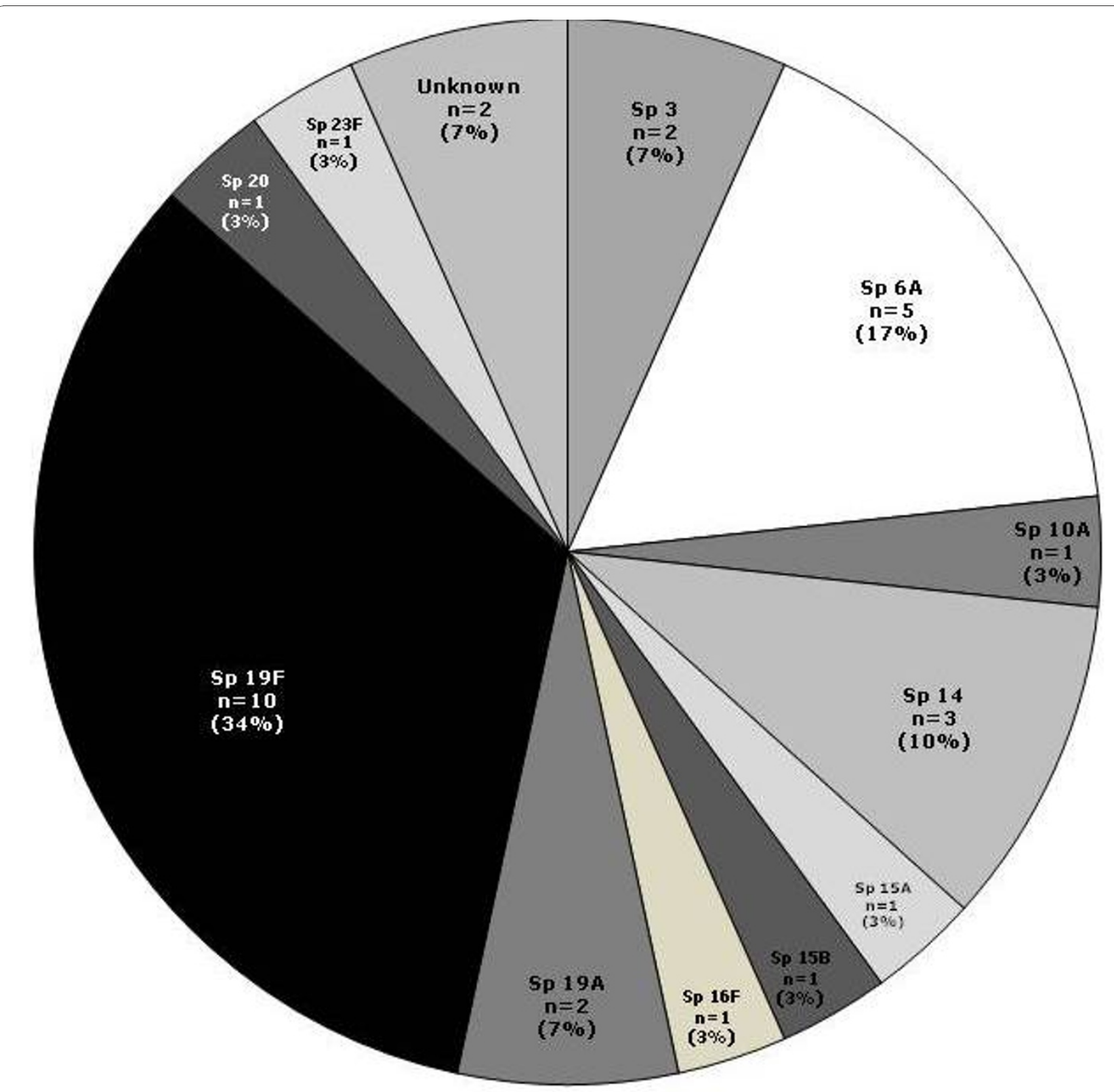

Figure 3 Serotype distribution of $S$. pneumoniae $(\mathrm{N}=30)$ positive isolates cultured from middle ear fluid samples. The pie chart shows each serotypes of S. pneumoniae with their respective percentage of positive episodes.

point estimate for all of the PCV7 serotypes was that of $19 \mathrm{~F}$, at $25 \%$ (95\% CI -14 to 51$)$ [21]. In this study, 3 and 19A together represented $13 \%$ of the pneumococcal AOM. Serotype 14 has previously been documented to be the most frequent in Colombia [20]. Similar to studies in Costa Rica [10,37] and Chile [11], 19F was the most frequently isolated pneumococcal serotype in this study, followed by $6 \mathrm{~A}$ and 14 . Global data shows the most common pneumococcal serotypes causing AOM are $3,6 \mathrm{~A}, 6 \mathrm{~B}, 9 \mathrm{~V}, 14,19 \mathrm{~A}, 19 \mathrm{~F}$ and $23 \mathrm{~F}$ [38] indicating that potential coverage for pneumococcal serotypes included in the available formulations ranged from $60-86 \%[37,39]$.

The pneumococcal serotypes targeted by $\mathrm{PCV}-7$ and PCV-13 comprise $47 \%$ (63\% if 6A cross-protection is assumed) and $76 \%$, respectively, of the pneumococci isolated from AOM samples in this study, and thus $14.1 \%$ (19.2\% assuming 6A cross-protection) and $23.2 \%$ of all AOM cases sampled in this study $[21,25]$. The pneumococcal serotypes targeted by PHiD-CV also 


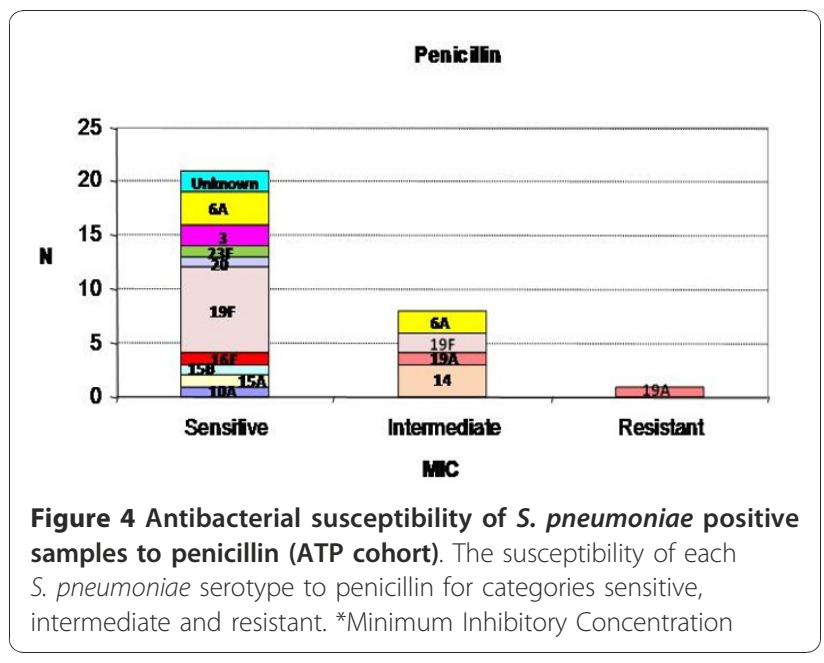

comprise $14.1 \%$ (19.2\% assuming 6A cross-protection) of all AOM cases sampled; in addition, PHiD-CV also targets $H$. influenzae which represents an additional $27 \%$ of all AOM cases sampled. It is important to note, however, that neither clinical efficacy nor effectiveness data

Table 3 Antibacterial susceptibility of samples positive for S. pneumoniae (ATP cohort)

\begin{tabular}{|c|c|c|c|c|}
\hline \multirow[t]{2}{*}{ Antibiotics } & \multicolumn{4}{|c|}{ Serotypes } \\
\hline & $\begin{array}{l}6 \mathrm{~A} n \\
(\%)\end{array}$ & $\begin{array}{l}14 \mathrm{n} \\
(\%)\end{array}$ & $\begin{array}{c}19 \mathrm{~A} \mathrm{n} \\
(\%)\end{array}$ & $\begin{array}{c}19 \mathrm{~F} \mathrm{n} \\
(\%)\end{array}$ \\
\hline Penicillin & $\begin{array}{l}3(60) \mathrm{S} \\
2(40) \mathrm{I}\end{array}$ & $\begin{array}{c}3(100) \\
1\end{array}$ & $\begin{array}{l}1(50) \text { I } \\
1(50) R\end{array}$ & $\begin{array}{l}8(80) \mathrm{S} \\
2(20) \mathrm{I}\end{array}$ \\
\hline Cefotaxime & $2(40) \mathrm{S}$ & $\begin{array}{l}1(33) \mathrm{S} \\
2(67) \mathrm{I}\end{array}$ & $1(50) R$ & $\begin{array}{l}7(70) \mathrm{S} \\
1(10) \text { I }\end{array}$ \\
\hline Erythromycin & $\begin{array}{l}3(60) \mathrm{S} \\
2(40) \mathrm{R}\end{array}$ & $\begin{array}{c}3(100) \\
5\end{array}$ & $\begin{array}{l}1(50) \mathrm{S} \\
1(50) \mathrm{R} \\
\end{array}$ & $\begin{array}{l}8(80) \mathrm{S} \\
2(20) \mathrm{R} \\
\end{array}$ \\
\hline Chloramphenicol & $\begin{array}{c}5(100) \\
5\end{array}$ & $\begin{array}{c}3(100) \\
5\end{array}$ & $2(100) S$ & $\begin{array}{c}10(100) \\
\mathrm{S} \\
\end{array}$ \\
\hline Tetracycline & $\begin{array}{c}5(100) \\
5 \\
\end{array}$ & $\begin{array}{l}2(67) \mathrm{S} \\
1(33) \mathrm{I} \\
\end{array}$ & $1(50) S, R$ & $\begin{array}{l}5(50) \mathrm{S} \\
5(50) \mathrm{R} \\
\end{array}$ \\
\hline Levofloxacin & $\begin{array}{c}5(100) \\
5\end{array}$ & $\begin{array}{c}3(100) \\
5\end{array}$ & $2(100) \mathrm{S}$ & $\begin{array}{c}10(100) \\
\mathrm{S}\end{array}$ \\
\hline $\begin{array}{l}\text { Trimethoprim/ } \\
\text { Sulfamethoxazole }\end{array}$ & $\begin{array}{l}4(80) S \\
1(20) R\end{array}$ & $\begin{array}{c}3(100) \\
R\end{array}$ & $1(50) S, R$ & $\begin{array}{l}6(60) \mathrm{S} \\
3(30) \mathrm{R} \\
1(10) \mathrm{I}\end{array}$ \\
\hline Other & $\begin{array}{c}5(100) \\
S\end{array}$ & $\begin{array}{c}3(100) \\
\mathrm{S}\end{array}$ & $1(100) \mathrm{S}$ & $9(100) \mathrm{S}$ \\
\hline
\end{tabular}

$\mathrm{n}=$ number of samples in a given category

$(\%)=n /$ Number of samples with results available ${ }^{*} 100$

$\mathrm{S}=$ sensitive; $\mathrm{I}=$ intermediate; $\mathrm{R}=$ resistant

NT $=$ not tested

Note: None of the serotypes are tested for Amoxicillin/Clavulanate and Cefuroxime; For serotype 6A, only 1 sample was sensitive for Azithromycin; For serotypes $15 \mathrm{~A}$ and $15 \mathrm{~B}$, only 1 sample was sensitive for each mentioned antibiotic; Serotype 3 had 2 samples sensitive for each antibiotic and Not Tested for Cefotaxime; Serotype 10A had 1 sample sensitive for all antibiotics; Serotype 20 had 1 sample sensitive for all antibiotics, Not Tested for Chloramphenicol and Missing for other; Serotype $16 \mathrm{~F}$ and $23 \mathrm{~F}$ had 1 sample resistant to Tetracycline, 1 sample Not Tested for Cefotaxime, 1 missing for Other and 1 sample sensitive for other antibiotics against $\mathrm{AOM}$ are available for either PHiD-CV or PCV13 , and so the magnitude of the clinical impact of each remains undetermined.

There were some limitations to the study. This study is limited by a small sample size which affects the precision of the estimates. In addition, the enrolled children were recruited from private centers which might not be representative of the entire Colombian population. However, we note that both pathogen and serotype distribution are similar to what has been seen in studies elsewhere.

\section{Conclusions}

In summary, $H$. influenzae (NTHi) and S. pneumoniae were the most commonly isolated bacteria in the study in all age groups. Vaccination could be an effective strategy in Colombia for preventing AOM disease. Considering the importance of $H$. influenzae as a causative agent of AOM, a conjugate vaccine with efficacy against both $H$. influenzae and S. pneumoniae could potentially prevent most bacterial AOM among Colombian children.

\section{List of abbreviations}

AOM: Acute Otitis Media; ATP: According-To-Protocol; Cl: Confidence Interval; CLSI: Clinical and Laboratory Standards Institute; GSK: GlaxoSmithKline; H. influenzae: Haemophilus influenzae; M. catarrhalis: Moraxella catarrhalis; MEF: Middle Ear Fluid; NTHi: Non-typeable Haemophilus influenzae;PCV: Pneumococcal Conjugate Vaccine; PHiD-CV: H. influenzae protein D conjugate vaccine; S. aureus: Staphylococcus aureus; S. pneumoniae: Streptococcus pneumoniae;S. pyogenes: Streptococcus pyogenes; SAS: Statistical Analysis System; SIREVA: Surveillance reports of the Regional System for Vaccines

\section{Acknowledgements}

We thank the infants and their families for participating in this trial. We also thank the GSK Colombia (CARICAM) team for contributing and supporting study co-ordination and monitoring activities for this study. The authors would like to thank Mohd. Najeeb Ashraf for providing assistance in medical writing, Gunasekaran Ramakrishnan for supporting with statistical analysis, Flavia Lamarao and Jessica Mattos for publication management and coordination (all employed by GSK Biologicals).

GSK Biologicals was the funding source and was involved in all stages of the study conduct and analysis. GSK Biologicals also took in charge all costs associated with the development and the publishing of the present publication. Synflorix is a trademark of the GlaxoSmithKline group of companies; Prevnar/Prevenar is a trademark of Pfizer/Wyeth, USA

\section{Author details}

${ }^{1}$ Centro de Estudios en Infectologia Pediatrica CEIP, Cali, Colombia. ${ }^{2}$ GlaxoSmithKline Biologicals, Panama City, Panama. ${ }^{3}$ GlaxoSmithKline Biologicals, Wavre, Belgium. ${ }^{4}$ GlaxoSmithKline Biologicals, Rio de Janeiro, Brazil.

\section{Authors' contributions}

All authors were involved at study conception and design stage and/or acquisition of data and interpretation of data; draft/critical revision of the article and final approval of the manuscript.

\section{Competing interests}

All investigators at study clinical sites were funded though their institutions to do the study.

Maria Mercedes Castrejon, Rodrigo DeAntonio, William P Hausdorff and Romulo E Colindres are employees of GlaxoSmithKline Biologicals, William P Hausdorff, Romulo E Colindres, Maria Mercedes Castrejon and Rodrigo DeAntonio have stock options. 
Pio Lopez received honoraria/paid expert testimony/travel grants from the commercial entity that sponsored the study.

Alexandra Sierra, Beatriz Vanegas and Mercedes Adriana Zapata declare no conflict of interest.

Received: 30 September 2010 Accepted: 5 January 2011

Published: 5 January 2011

\section{References}

1. Kathy C, Mathew W: Otitis media, ear, nose and throat. eMedicine 2005, 1-11.

2. Brobby GW: The discharging ear in the tropics: a guide to diagnosis and management in a district hospital. Trop Doct 1992, 22:10-13.

3. Valenzuela MT, O' Loughlin R, De La Hoz F, Gomez E, Constenla D, Sinha A, Valencia JE, Flannery B, De Quadros CA: The burden of pneumococcal disease among Latin American and Caribbean children: review of the evidence. Rev Panam Salud Publica/Pan Am J Public Health 2009. 25:270-279.

4. Teele DW, Klein JO, Rosner B: Epidemiology of otitis media during the first seven years of life in children in greater Boston: a prospective, cohort study. J Infect Dis 1989, 160:83-94.

5. Liese J, Carmona A, Cantarutti L, Silfverdal SA, Fuat A, Vollmar J, Pircon JY, Rosenlund M: Incidence of acute otitis media in young children seen in European medical practices [abstract]. Presented at: 6th World Congress of the World Society for Pediatric Infectious Diseases (WSPID) 2009, Source: MIS (5564702)

6. Trujillo H, Callejas R, Mejía Gl, Castrillon L: Bacteriology of middle ear fluid specimens obtained by tympanocentesis from 111 Colombian children with acute otitis media. Pediatr Infect Dis J 1989, 8:361-363.

7. Heikkinen T, Chonmaitree T: Importance of respiratory viruses in acute otitis media. Clin Microbiol Rev 2003, 16:230-241.

8. Bluestone C: Definitions, Terminology and Classification. In Evidence-Based Otitis Media. Edited by: Rosenfeld RM, Bluestone CD. Hamilton; Saint Louis: BC Decker; 1999:85-103.

9. Leibovitz E, Jacobs MR, Dagan R: Haemophilus influenzae: a significant pathogen in acute otitis media. Pediatr Infect Dis J 2004, 23:1142-1152.

10. Arguedas A, Dagan R, Soley C, Loaiza C, Knudsen K, Porat N, Perez A, Brilla E, Herrera ML: Microbiology of otitis media in Costa Rican children, 1999 through 2001. Pediatr Infect Dis J 2003, 22:1063-1068.

11. Rosenblut A, Santolaya ME, Gonzalez P, Corbalan V, Avendano LF, Martinez MA, Hormazabal JC: Bacterial and viral etiology of acute otitis media in Chilean children. Pediatr Infect Dis J 2001, 20:501-507.

12. Pichichero ME: Evolving shifts in otitis media pathogens: relevance to a managed care organization. AJMC 2005, 11(6:Suppl):201

13. Casey JR, Adlowitz DG, Pichichero ME: New patterns in the otopathogens causing acute otitis media six to eight years after introduction of pneumococcal conjugate vaccine. Pediatr Infect Dis J 2010, 29:304-309.

14. Evridiki KV, Drosos EK, Maria SK, Anastasios MK, Matthew EF: Antibiotics versus placebo or watchful waiting for acute otitis media: a metaanalysis of randomized controlled trials. J Antimicrob Chemother 2009, 64:16-24.

15. Rovers MM, Schilder AG, Zielhuis GA, Rosenfeld RM: Otitis media. Lancet 2004, 363:465-473.

16. Riquelme PM, Rincon VP, Garcia RL, Tusset CJ, Bravo TR, Fernandez PC: Acute otitis media in a pediatric primary care unit. An Pediatr (Barc) 2004, 61:408-412.

17. Williams RL, Chalmers TC, Stange KC, Chalmers FT, Bowlin SJ: Use of antibiotics in preventing recurrent acute otitis media and in treating otitis media with effusion. A meta-analytic attempt to resolve the brouhaha. JAMA 1993, 270:1344-1351.

18. Centers for Disease Control and Prevention: Pneumococcal disease. In Epidemiology and prevention of vaccine-preventable diseases: the pinkbook. Volume 2007. 10 edition. Centers for Disease Control and Prevention;257-270.

19. Camargos P, Fischer GB, Mocelin H, Dias C, Ruvinsky R: Penicillin resistance and serotyping of Streptococcus pneumoniae in Latin America. Pediatr Respir Rev 2006, 7:209-214.

20. Garcia S, Levine OS, Cherian T, Gabastou JM, Andrus J, Working Group members: Pneumococcal disease and vaccination in the Americas: an agenda for accelerated vaccine introduction. Rev Panam Salud Publica 2006, 19:340-348.

21. Eskola J, Kilpi T, Palmu A, Jokinen J, Haapakoski J, Herva E, Takala A, Kayhty H, Karma P, Kohberger R, Siber G, Makela PH: Efficacy of a pneumococcal conjugate vaccine against Acute Otitis Media. N Engl $J$ Med 2001, 344:403-409.

22. Black S, Shinefield H, Fireman B, Lewis E, Ray P, Hansen JR, Elvin L, Ensor KM, Hackell J, Siber G, Malinoski F, Madore D, Chang I, Kohberger R, Watson W, Austrian R, Edwards K: Efficacy, safety and immunogenicity of heptavalent pneumococcal conjugate vaccine in children. Northern California Kaiser Permanente Vaccine Study Center Group. Pediatr Infect Dis J 2000, 19:187-195.

23. Block SL, Hedrick J, Harrison CJ, Tyler R, Smith A, Findlay R, Keegan E: Community-wide vaccination with the heptavalent pneumococcal conjugate significantly alters the microbiology of Acute Otitis Media. Pediatr Infect Dis J 2004, 23:829-833.

24. Casey JR, Pichichero ME: Changes in frequency and pathogens causing Acute Otitis Media in 1995-2003. Pediatr Infect Dis J 2004 23:824-828.

25. Prymula R, Peeters P, Chrobok V, Kriz P, Novakova E, Kaliskova E, Kohl I, Lammel P, Poolman J, Prieels JP, Schuerman L: Pneumococcal capsular polysaccharides conjugated to protein $D$ for prevention of acute otitis media caused by both Streptococcus pneumoniae and non-typable Haemophilus influenzae: a randomised double-blind efficacy study. Lancet 2006, 367:740-748

26. Arguedas A, Dagan R, Guevara S, Porat N, Soley C, Perez A, Brilla R: Middle ear fluid Streptococcus pneumoniae serotype distribution in Costa Rican children with otitis media. Pediatr Infect Dis J 2005, 24:631-634.

27. Hyden D, Akerlind B, Peebo M: Inner ear and facial nerve complications of acute otitis media with focus on bacteriology and virology. Acta Otolaryngol 2006, 126:460-466.

28. Commisso R, Romero-Orellano F, Montanaro PB, Romero-Moroni F, RomeroDiaz R: Acute otitis media: Bacteriology and bacterial resistance in 205 pediatric patients. Int I Pediatr Otorhinolaryngol 2000, 56:23-31.

29. Alvarado O, Aguilar L, Soley C, Loaiza C, Guevara S, Rincon G, Arguedas J, Arguedas A: Microbiology of otitis media in Costa Rican children between 2002-2007 [Abstract G-982]. Presented at: 47th Annual Interscience Conference on Antimicrobial Agents and Chemotherapy (ICAAC) 2007.

30. Parras MM, Martinez AG, Echaniz-Aviles G, Lazcano-Ponce E, Apolinar YC, Colindres R, Pircon JY, Hausdorff W: Bacterial etiology and serotypes of Acute Otitis Media in children in Mexico [Abstract no: A-181-001000611]. Presented at: 6th World Congress of the World Society for Pediatric Infectious Diseases (WSPID), Buenos Aires, Argentina, November 2009, Source: MIS (5564557).

31. Murphy TF, Faden H, Bakaletz LO, Kyd MJ, Forsgren A, Campos J, Virji M Pelton SI: Nontypeable Haemophilus influenzae as a Pathogen in Children. Pediatr Infect Dis J 2009, 28:43-48.

32. Broides A, Dagan R, Greenberg D, Givon-Lavi N, Leibovitz E: Acute Otitis Media caused by Moraxella catarrhalis: Epidemiologic and Clinical Characteristics. Clin Infect Dis 2009, 49:1641-1647.

33. Turner D, Leibovitz, Aran A, Piglansky L, Raiz S, Leiberman A, Dagan R: Acute otitis media in infants younger than two months of age: microbiology, clinical presentation and therapeutic approach. Pediatr Infect Dis J 2002, 21:669-674.

34. Valenzuela MT, de Quadros C: Antibiotic resistance in Latin America: A cause of alarm. Vaccine 2009, 27S:C25-C28.

35. Bluestone CD, Stephenson JS, Martin LM: Ten-year review of otitis media pathogens. Pediatr Infect Dis J 1992, 11(suppl):S7-S11.

36. Piglansky L, Leibovitz E, Raiz S, Greenberg D, Press J, Leiberman A, Dagan R: Bacteriologic and clinical efficacy of high dose amoxicillin for therapy of acute otitis media in children. Pediatr Infect Dis J 2003, 22:405-413.

37. Arguedas A, Dagan R, Guevara S, Porat N, Soley C, Perez A, Brilla R: Middle ear fluid Streptococcus pneumoniae serotype distribution in Costa Rican children with otitis media. Pediatr Infect Dis J 2005, 24:631-634

38. Rodgers GL, Arguedas A, Cohen R, Dagan R: Global serotype distribution among Streptococcus pneumoniae isolates causing otitis media in children: Potential implications for pneumococcal conjugate vaccines. Vaccine 2009, 27:3802-3810. 
39. Hausdorff WP, Yothers G, Dagan R, Kilpi T, Pelton SI, Cohen R, Jacobs MR, Kaplan SL, Levy C, Lopez EL, Mason EO Jr, Syriopoulou V, Wynne B, Bryant J: Multinational study of pneumococcal serotypes causing acute otitis media in children. Pediatr Infect Dis J 2002, 21:1008-1016

\section{Pre-publication history}

The pre-publication history for this paper can be accessed here: http://www.biomedcentral.com/1471-2334/11/4/prepub

doi:10.1186/1471-2334-11-4

Cite this article as: Sierra et al:: Non-typeable Haemophilus influenzae and Streptococcus pneumoniae as primary causes of acute otitis media in colombian children: a prospective study. BMC Infectious Diseases 2011 11:4.

Submit your next manuscript to BioMed Central and take full advantage of:

- Convenient online submission

- Thorough peer review

- No space constraints or color figure charges

- Immediate publication on acceptance

- Inclusion in PubMed, CAS, Scopus and Google Scholar

- Research which is freely available for redistribution 